Check for updates

Cite this: Phys. Chem. Chem. Phys., 2018, 20, 30054

DOI: $10.1039 / c 8 c p 91924 k$

\section{Correction: Conceptual design and analysis of ITM oxy-combustion power cycles}

\author{
Andrea König, (D) ${ }^{a}$ Nick D. Mancini† ${ }^{b}$ and Alexander Mitsos (DD $\star^{b}$ \\ Correction for 'Conceptual design and analysis of ITM oxy-combustion power cycles' by N. D. Mancini et al., \\ Phys. Chem. Chem. Phys., 2011, 13, 21351-21361.
}

rsc.li/pccp

In ref. 1 Mancini and Mitsos simulated a variety of ion transport membrane (ITM) power cycles. The authors discussed the benefits of partial emissions cycles over a combination of zero-emissions cycles and conventional combined cycles using a linear combination metric.

Ref. 1 presented the linear combination of the zero-emissions cycle and conventional combined cycle as a line in a graph, i.e., Fig. 8 of ref. 1 with First Law Efficiency and $\mathrm{CO}_{2}$ emissions as evaluation criteria. Ref. 1 thus implied that the First Law Efficiency of a linear combination of non-hybrid plants can be determined by simple linear interpolation of the efficiencies of each non-hybrid plant, i.e.,

$$
\eta_{\text {lincom,incorrect }}=\sum_{i} \lambda_{i} \cdot \eta_{i}=\sum_{i} \frac{\dot{W}_{\text {out }, i}}{\dot{W}_{\text {out }, \text { overall }}} \cdot\left(\frac{\dot{W}_{\text {out }, i}}{\mathrm{LHV} \cdot \dot{n}_{\text {fuel }, i}}\right),
$$

with $\lambda$ being the plant split fraction, which is based on the output of each cycle type, $i$,

$$
\lambda_{i}=\frac{\dot{W}_{\text {out }, i}}{\dot{W}_{\text {out }, \text { overall }}} .
$$

It can be seen that $\eta_{\text {lincom,incorrect }}$ is not equivalent to the overall First Law Efficiency, $\eta_{\text {overall }}=\frac{\dot{W}_{\text {out,overall }}}{\dot{n}_{\text {fuel,overall }} \cdot \text { LHV }}$ Instead, one should use a reverse linear interpolation. Then $\dot{W}_{\text {out }, i}$ is canceled out, such that the overall First Law Efficiency of the linear combination is correctly determined,

$$
\begin{aligned}
\eta_{\text {lincom,correct }} & =\frac{1}{\sum_{i} \lambda_{i} \cdot \frac{1}{\eta_{i}}}=\frac{1}{\sum_{i} \frac{\dot{W}_{\text {out }, i}}{\dot{W}_{\text {out }, \text { overall }}} \cdot \frac{\dot{n}_{\text {fuel }, \mathrm{i}} \cdot \mathrm{LHV}}{\dot{W}_{\text {out }, \mathrm{i}}}} \\
& =\frac{\dot{W}_{\text {out }, \text { overall }}}{\sum_{i} \dot{n}_{\text {fuel }, \mathrm{i}} \cdot \mathrm{LHV}}=\frac{\dot{W}_{\text {out }, \text { overall }}}{\dot{n}_{\text {fuel,overall }} \cdot \mathrm{LHV}}=\eta_{\text {overall }} \cdot
\end{aligned}
$$

Ref. 1 correctly used a linear interpolation for the second performance criterion, i.e., $\mathrm{CO}_{2}$ emissions. This leads to an inverse relationship of the two performance criteria, i.e.,

$$
\left(\mathrm{CO}_{2} \text { emissions }\right)_{i} \propto \lambda_{i} \propto \frac{1}{\eta_{i}} .
$$

Based on this insight, Fig. 1 shows how the performance line of Fig. 8 in ref. 1 has to be amended.

\footnotetext{
${ }^{a}$ Aachener Verfahrenstechnik - Process Systems Engineering, RWTH Aachen University, Forckenbeckstr. 51, 52074 Aachen, Germany

${ }^{b}$ Department of Mechanical Engineering, Massachusetts Institute of Technology, 77 Massachusetts Avenue (MIT 3-158), Cambridge, MA 02139, USA.

E-mail: amitsos@alum.mit.edu; Fax: +1 (617)-258-5802; Tel: +1 (617)-324-6768

$\dagger$ Present address: Tesla Inc., 3500 Deer Creek Rd., Palo Alto, CA 94304, USA.
} 


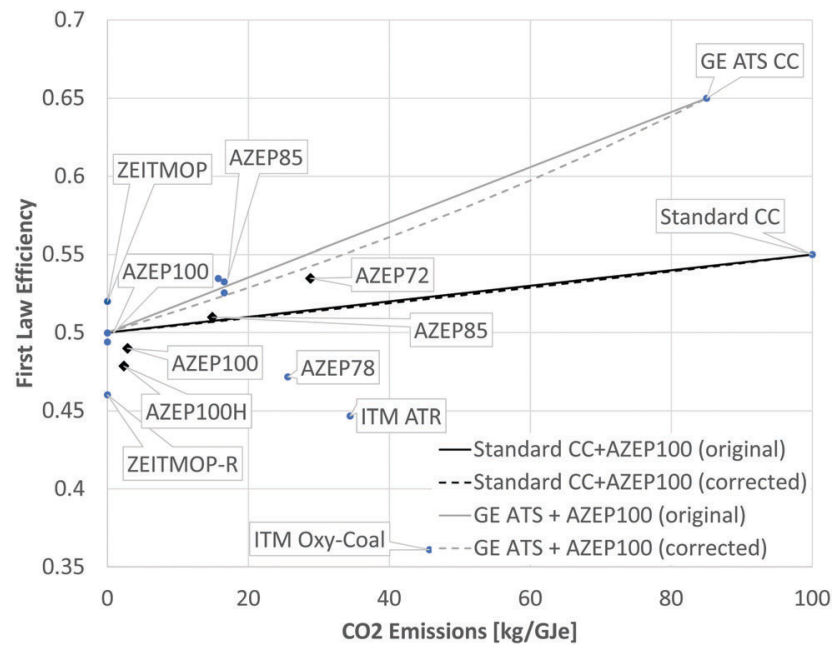

Fig. 1 Corrected linear combination originally from ref. 1.

When comparing the corrected function to the line originally illustrated in ref. 1 it becomes clear that the actual performance of a linear combination is slightly worse than previously indicated. Thus, from a relative perspective, partial emission cycles perform slightly better than previously thought.

In conclusion, the application of the linear combination in ref. 1 is incorrect but results in very small numerical errors. The Royal Society of Chemistry apologises for these errors and any consequent inconvenience to authors and readers.

\section{References}

1 N. D. Mancini and A. Mitsos, Phys. Chem. Chem. Phys., 2011, 13, 21351-21361. 